\title{
Wild fish aggregations around fish farms in the Gulf of Aqaba, Red Sea: implications for fisheries management and conservation
}

\author{
Aytaç Özgül ${ }^{1}$, Dror Angel ${ }^{2, *}$ \\ ${ }^{1}$ Faculty of Fisheries, Ege University, 35100 Bornova-Izmir, Turkey \\ ${ }^{2}$ Charney School of Marine Science, University of Haifa, Mount Carmel, 31905 Haifa, Israel
}

\begin{abstract}
Fish farm structures attract a variety of fish species by providing solid structure, and 'free meals' in the form of uneaten feed and feces that fall from the cages. In this study, we compared the composition and abundances of wild fish populations around 2 fish farms in the Red Sea and at nearby reference locations. Fish assemblages were evaluated by SCUBA divers carrying out rapid visual censuses in August, September and October 2007. A total of 87238 fishes, representing 39 species and 25 families and a number of trophic levels, were observed. Overall, the abundance, biomass, and diversity of wild fish were much greater at the sea cages than at the open-water reference sites, at both fish farms. It is noteworthy that 35 out of the 39 species observed at the farms were juveniles and adults of coral-reef fish species. This finding is especially interesting, considering that the nearest coral reefs were $>4 \mathrm{~km}$ away and reef fishes generally have small home ranges, typically $<1 \mathrm{~km}$. Among these, the Carangidae (5 species), Sparidae (3 species), and Pomacentridae ( 3 species) contributed the most to species richness. In light of strong fishing pressures in many areas, the large populations of wild fishes at the fish farms, the high availability of nourishment from artificial food pellets, and restrictions on fishing within farm lease areas, we suggest that coastal net-cage fish farms may serve as small marine protected areas.
\end{abstract}

KEY WORDS: Abundance $\cdot$ Visual census $\cdot$ Wild fishes $\cdot$ Red Sea $\cdot$ Aquaculture $\cdot$ Spatial-temporal variability $\cdot$ Impact

\section{INTRODUCTION}

Since the development of modern sea-cage aquaculture in the early 1980 s, the number of net-cage fish farms has increased rapidly throughout the coastal areas of the world (Theodorou 1999, Ferlin \& La Croix 2000, Sanchez-Mata \& Mora 2000, Beveridge 2004, Halwart et al. 2007, Bostock et al. 2010). Total reported cage production of finfish amounted to 3.4 million tonnes $\mathrm{yr}^{-1}$ in 2010 (FAO 2012).

Natural and artificial fish aggregation devices (FADs) in the marine environment are widely recognized for their capacity to attract pelagic fishes (Hunter \& Mitchell 1967, Deudero et al. 1999,

\footnotetext{
${ }^{*}$ Corresponding author. Email: adror@research.haifa.ac.il
}

Freon \& Dagorn 2000). Sea-cage fish farms attract wild fish by providing structure in the pelagic environment, and uneaten feed and feces that fall through the cages may enhance the attractive effect (Phillips et al. 1985, Bjordal \& Skar 1992, Dempster et al. 2002). Fish farms may therefore affect the presence, abundance, diet, and residence times of fishes in a given area (Carss 1990). Aquaculture facilities in the marine environment that serve as FADs show characteristics of healthy artificial reefs, and as such may help improve ecosystem and aquaculture management strategies (Costa-Pierce \& Bridger 2002, Alston et al. 2006, Benetti et al. 2006).

() The authors 2013. Open Access under Creative Commons by Attribution Licence. Use, distribution and reproduction are unrestricted. Authors and original publication must be credited. 
Aggregations of wild fishes are a global feature of sea-cage farms and were first documented by Carss (1990) around salmonid cages in Scotland. The phenomenon is ubiquitous, and large aggregations of pelagic and/or demersal fish have since been described around sea cages in a variety of locations, including Spain (Dempster et al. 2002), Greece (Smith et al. 2003, Thetmeyer et al. 2003), the Canary Islands (Boyra et al. 2004, Tuya et al. 2005), Norway (Bjordal \& Skar 1992), the Adriatic Sea (Bubic et al. 2011), Turkey (Akyol \& Ertosluk 2010), Indonesia (Sudirman et al. 2009), and Australia (Dempster et al. 2004, Felsing et al. 2005). Despite a growing literature on the occurrence of wild fishes around sea cages, only a few studies (including Angel \& Spanier 2002, Angel et al. 2002, Golani 2003, Golani et al. 2008) have examined the wild fish populations near fish farms in the Red Sea. These studies suggest that the fish assemblages associated with the farms are a lot more similar to coral-reef fish communities than to local pelagic communities, despite considerable distance between farms and reefs. Coral-reef fishes generally have small home ranges (Ogden \& Quinn 1984, Zeller at al. 2003), yet the distance between the fish farms and the closest coral reefs was $>4 \mathrm{~km}$, a range in excess of that recorded for most reef species.

In the present study, we evaluated species composition and total aggregated abundance and biomass of wild fish at 2 fish farms in the Red Sea and at reference locations in an effort to describe and understand the role of fish farms in structuring the wild fish assemblage in tropical environments.

\section{MATERIALS AND METHODS}

\section{Study area and fish farms}

This study was conducted at the fish farms 'Ardag' and 'Dagsuf', situated at the northern tip of the Gulf of Aqaba, near the Jordan-Israel border, from August to October 2007 (Fig. 1). The Gulf of Aqaba, Red Sea, supports a rich and highly diverse community of tropical and subtropical invertebrates and fishes, in spite of its generally oligotrophic waters and narrow range of water temperatures (annual range: 21 to $27^{\circ} \mathrm{C}$; Reiss \& Hottinger 1984). The diversity of fish and invertebrate species that characterizes the coral reefs and a host of other natural features make the gulf an increasingly popular tourist destination. Eilat's coral reefs are threatened by various anthropogenic activities, such as a port and phosphate terminal, oil pipeline, mariculture, municipal sewage, the municipal marina, and tourism (Zakai \& Chadwick-Furman 2002, Atkinson et al. 2004).

The farms we observed reared mainly gilthead seabream Sparus aurata. The farms were established in 1989 (Ardag) and 1993 (Dagsuf), and both were dismantled in June 2008, in accordance with a government decision. The fish farms consisted of 2 to 3 parallel sets of fish cages, and in the last decade of their operation, overall annual production ranged from 2000 to $3000 \mathrm{t}$. Specific details for each fish farm are given in Table 1.

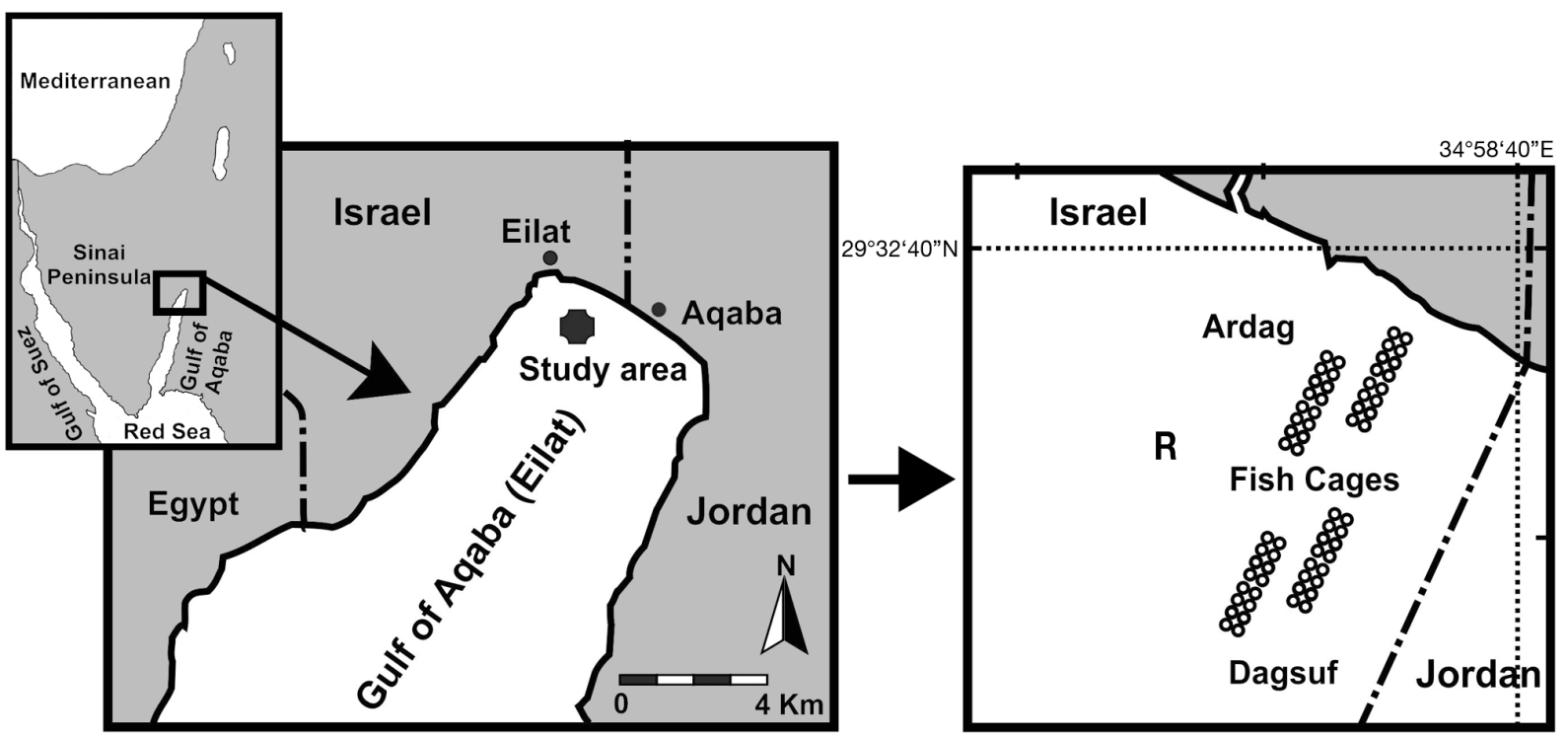

Fig. 1. Location of the study area at the northern end of the Gulf of Aqaba, Red Sea. The sandy seafloor in this area has few solid substrates (e.g. concrete moorings or debris associated with the fish farms, shown in Fig. 2) and there were no coral reefs near the fish farms. ' $\mathrm{R}$ ' indicates the location of the reference station, ca. $500 \mathrm{~m}$ west of the fish farms 
Table 1. Physical and environmental features of the net-cage fish farms. HDPE: high-density polyethylene

\begin{tabular}{|lcccccc|}
\hline Fish farm & Location & $\begin{array}{c}\text { Distance from } \\
\text { shore (m) }\end{array}$ & $\begin{array}{c}\text { Year established- } \\
\text { Shut down }\end{array}$ & $\begin{array}{c}\text { Seafloor depth } \\
\text { range (m) }\end{array}$ & $\begin{array}{c}\text { Maximum no. } \\
\text { of cages }\end{array}$ & $\begin{array}{c}\text { Cage } \\
\text { material }\end{array}$ \\
\hline Ardag & $\begin{array}{l}29^{\circ} 32^{\prime} 28^{\prime \prime} \mathrm{N}, \\
34^{\circ} 58^{\prime} 27^{\prime \prime} \mathrm{E}\end{array}$ & 300 & $1989-2008$ & $19-30$ & 54 \\
Dagsuf & $\begin{array}{l}29^{\circ} 32^{\prime} 23^{\prime \prime} \mathrm{N}, \\
34^{\circ} 58^{\prime} 22^{\prime \prime} \mathrm{E}\end{array}$ & 600 & $1993-2008$ & $32-46$ & 45 & HDPE \\
\hline
\end{tabular}

\section{Rapid visual counts}

At each of the 2 fish farms, wild fish around the cages were counted on 3 randomly selected days over a period of 2 mo using a rapid non-destructive underwater visual census method that allowed for a high degree of replication (Dempster et al. 2002). Six 5 min census counts were conducted on each date within each farm complex. An equal number of counts (6) were performed at a reference location, situated $\sim 500 \mathrm{~m}$ to the west of both farms, beyond the borders of the lease. All counts were made between 11:00 and 14:00 h. Each count covered a volume of approximately $11250 \mathrm{~m}^{3}$ (15 m wide $\times 15 \mathrm{~m}$ deep $\times$ $50 \mathrm{~m}$ long), and was conducted at depths of 5 to $12 \mathrm{~m}$, allowing for the water column from the surface to the bottom of the adjacent cage to be scanned effectively (Fig. 2). Each count was made by 2 divers. The first diver concentrated on estimating the abundances of the dominant species present. Fish were counted in abundance groups of $1,2-5,6-10,11-30,31-50$, 51-100, 101-200, 201-500, 501-1000, and >1000 to minimize error, based on the method of HarmelinVivien et al. (1985), and the average total length of each species was noted. The second diver followed slightly behind the first and specifically looked for both highly mobile species and smaller, less obvious fish that may have been missed by the first diver (Dempster et al. 2002, 2005).

Prior to the census, the various species of fish were identified visually by divers and documented by underwater photography. Conversions to biomass were made for each species based on estimated lengths and published length-weight relationships from FishBase (Froese \& Pauly 2012). Seawater temperature (measured using a dive computer, SuuntoStinger) and horizontal Secchi-disc readings (to estimate visibility) were recorded on days when census counts were conducted.

\section{Multivariate analysis}

Fish abundance data for each fish farm were analyzed using non-metric multidimensional scaling (nMDS) (Clarke 1993) to assess differences in community structure among the farms and reference locations for each sampling time. Abundance indices

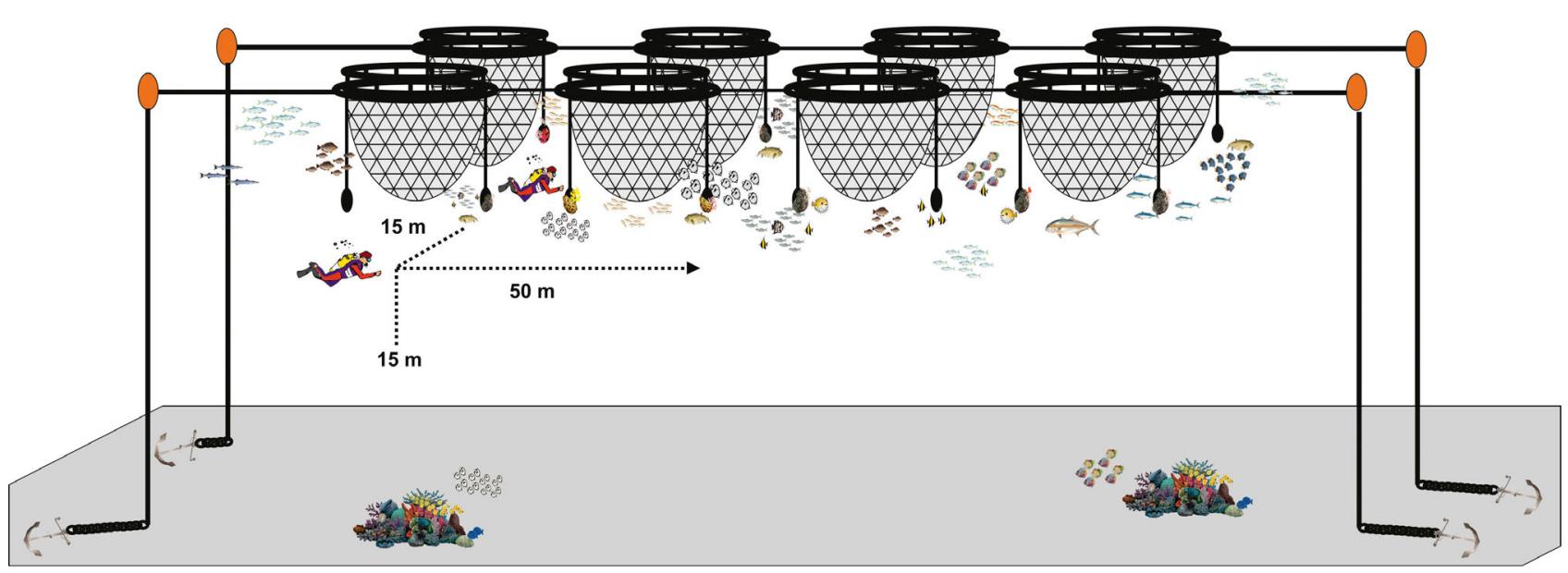

Fig. 2. Sketch showing 2 divers carrying out the underwater rapid visual census around fish cages at depths of 5 to $12 \mathrm{~m}$, with the water column scanned from surface to bottom of adjacent cage (extension of census volume indicated). The sandy seafloor in this area has few solid substrates (e.g. concrete moorings or debris associated with the fish farms, shown in the sketch) and there were no coral reefs near the fish farms 
(Clarke \& Warwick 1994) and the results were presented as 2-dimensional plots. The analysis of similarity (ANOSIM) permutation test was used to assess the significance of differences between locations and among times within locations (Clarke 1993). The similarity percentage (SIMPER) procedure for the abundance data pooled over time was used to identify the contribution of individual species to differences between locations for each farm. All multivariate analyses were carried out using the PRIMER ${ }^{\complement}$ statistical package (Clarke \& Warwick 1994).

\section{Univariate analysis}

To examine the differences between the farm and reference sites, in terms of total abundances and biomass of aggregated wild fish, we used ANOVA and tested the factors Impact/Reference, Location, and Times initially. However, due to a majority of 0 values at reference sites, this analysis was considered unnecessary, and we simplified the design to the factors Location and Times nested in Location.
Prior to ANOVA, heterogeneity of variance was tested with Levene's test. Data were square-root $(x+1)$-transformed if variances were significantly different at $p \leq 0.05$, and $\ln (x+1)$-transformed if variances were still heterogeneous. When variance remained heterogeneous, untransformed data were analyzed, as ANOVA is robust to heterogeneity of variances, particularly for large balanced experiments (Underwood 1997). All univariate statistical analyses of data were performed using the SPSS 15.0 statistical package.

\section{RESULTS}

\section{Abundance}

We counted a total of 87238 fishes, representing 39 species and 25 families, at both sea-cage farms and nearby reference sites. Twenty-nine species were observed only at the fish cages, 4 species occurred at both farm and reference sites, and 5 species were seen only at the reference sites (Table 2). Of the 39 fish species recorded, 35 were reef-associated spe-

Table 2. Abundance (mean \pm SE) and body length (or range of lengths) of wild fish species for the 3 sampling dates at the fish farms and reference site. Values are from 18 rapid visual counts. Blank: not present

\begin{tabular}{|c|c|c|c|c|c|c|}
\hline \multirow[t]{2}{*}{ Fish } & \multicolumn{2}{|l|}{ Ardag } & \multicolumn{2}{|l|}{ Dagsuf } & \multicolumn{2}{|c|}{ Reference site } \\
\hline & $\begin{array}{l}\text { Abundance } \\
\text { (ind. } 11250 \mathrm{~m}^{-3} \text { ) }\end{array}$ & $\begin{array}{l}\text { Length } \\
\text { (cm) }\end{array}$ & $\begin{array}{c}\text { Abundance } \\
\text { (ind. } 11250 \mathrm{~m}^{-3} \text { ) }\end{array}$ & $\begin{array}{l}\text { Length } \\
\text { (cm) }\end{array}$ & $\begin{array}{c}\text { Abundance } \\
\text { (ind. } 11250 \mathrm{~m}^{-3} \text { ) }\end{array}$ & $\begin{array}{l}\text { Length } \\
\text { (cm) }\end{array}$ \\
\hline \multicolumn{7}{|l|}{ Actinopterygii } \\
\hline \multicolumn{7}{|l|}{ Atheriniformes } \\
\hline \multicolumn{7}{|l|}{ Atherinidae } \\
\hline Atherinomorus lacunosus & $13.89 \pm 13.89$ & 3 & $61.11 \pm 61.11$ & 3 & & \\
\hline \multicolumn{7}{|l|}{ Beloniformes } \\
\hline \multicolumn{7}{|l|}{ Belonidae } \\
\hline Tylosurus choram & & & $13.33 \pm 7.80$ & $10-15$ & & \\
\hline \multicolumn{7}{|l|}{ Hemiramphidae } \\
\hline Hyporhamphus gamberur & $30.89 \pm 19.47$ & $15-25$ & $34.44 \pm 27.25$ & $15-18$ & & \\
\hline \multicolumn{7}{|l|}{ Perciformes } \\
\hline \multicolumn{7}{|l|}{ Acanthuridae } \\
\hline Ctenochaetus striatus & $0.72 \pm 0.61$ & $6-14$ & & & & \\
\hline \multicolumn{7}{|l|}{ Apogonidae } \\
\hline Apogon fleurieu & $1.67 \pm 1.67$ & 5 & & & & \\
\hline \multicolumn{7}{|l|}{ Blenniidae } \\
\hline Petroscirtes mitratus & $0.06 \pm 0.06$ & 8 & $1.11 \pm 0.58$ & $6-10$ & & \\
\hline \multicolumn{7}{|l|}{ Caesionidae } \\
\hline Caesio suevica & $58.39 \pm 19.08$ & $15-23$ & $110.17 \pm 45.42$ & $15-23$ & $0.67 \pm 0.67$ & 20 \\
\hline \multicolumn{7}{|l|}{ Carangidae } \\
\hline Atule mate & $700.28 \pm 188.34$ & $8-12$ & $1560.83 \pm 191.52$ & $10-15$ & & \\
\hline Carangoides ferdau & & & $0.11 \pm 0.11$ & 25 & & \\
\hline Carangoides fulvoguttatus & & & $0.83 \pm 0.83$ & 80 & & \\
\hline Carangoides plagiotaenia & $0.28 \pm 0.28$ & 18 & & & & \\
\hline Decapterus macarellus & $104.11 \pm 56.53$ & $8-32$ & $3.56 \pm 1.88$ & $12-35$ & $22.22 \pm 22.22$ & 12 \\
\hline \multicolumn{7}{|l|}{ Chaetodontidae } \\
\hline Chaetodon fasciatus & $0.83 \pm 0.83$ & 5 & & & & \\
\hline Heniochus diphreutes & & & & & $0.33 \pm 0.33$ & 15 \\
\hline
\end{tabular}


Table 2 (continued)

\begin{tabular}{|c|c|c|c|c|c|c|}
\hline \multirow[t]{2}{*}{ Fish } & \multicolumn{2}{|c|}{ Ardaq } & \multicolumn{2}{|c|}{ _ Dagsuf } & \multicolumn{2}{|c|}{ _ Reference site } \\
\hline & $\begin{array}{c}\text { Abundance } \\
\text { (ind. } 11250 \mathrm{~m}^{-3} \text { ) }\end{array}$ & $\begin{array}{l}\text { Length } \\
\text { (cm) }\end{array}$ & $\begin{array}{l}\text { Abundance } \\
\text { (ind. } 11250 \mathrm{~m}^{-3} \text { ) }\end{array}$ & $\begin{array}{l}\text { Length } \\
\text { (cm) }\end{array}$ & $\begin{array}{c}\text { Abundance } \\
\text { (ind. } 11250 \mathrm{~m}^{-3} \text { ) }\end{array}$ & $\begin{array}{l}\text { Length } \\
\text { (cm) }\end{array}$ \\
\hline \multicolumn{7}{|l|}{ Cirrhitidae } \\
\hline Cirrhitichthys oxycephalus & & & $0.06 \pm 0.06$ & 5 & & \\
\hline \multicolumn{7}{|l|}{ Labridae } \\
\hline Thalassoma lunare & & & $0.06 \pm 0.06$ & 30 & & \\
\hline Thalassoma rueppellii & $1.22 \pm 0.68$ & $6-16$ & $0.83 \pm 0.45$ & $6-16$ & & \\
\hline \multicolumn{7}{|l|}{ Mullidae } \\
\hline Mulloides flavolineatus & & & & & $1.28 \pm 1.11$ & $12-15$ \\
\hline \multicolumn{7}{|l|}{ Nemipteridae } \\
\hline Scolopsis ghanam & & & & & $0.06 \pm 0.06$ & 15 \\
\hline \multicolumn{7}{|l|}{ Pomacentridae } \\
\hline Abudefduf vaigiensis & $35.33 \pm 15.00$ & $5-10$ & $10.56 \pm 3.52$ & $5-10$ & & \\
\hline Neopomacentrus miryae & $965.00 \pm 189.48$ & $6-8$ & $585.00 \pm 130.11$ & $6-8$ & $11.11 \pm 11.11$ & 4 \\
\hline Pomacentrus trichourus & & & & & $0.28 \pm 0.28$ & 8 \\
\hline \multicolumn{7}{|l|}{ Scombridae } \\
\hline Auxis rochei rochei & $0.11 \pm 0.11$ & 40 & & & & \\
\hline Scomber japonicus & & & $97.22 \pm 51.84$ & $23-26$ & & \\
\hline \multicolumn{7}{|l|}{ Serranidae } \\
\hline Anthias taeniatus & $5.06 \pm 4.43$ & $6-8$ & & & & \\
\hline Pseudanthias squamipinnis & $15.56 \pm 7.25$ & $5-8$ & $3.17 \pm 1.91$ & $5-8$ & $1.06 \pm 1.06$ & 8 \\
\hline \multicolumn{7}{|l|}{ Siganidae } \\
\hline Siganus rivulatus & $137.94 \pm 33.86$ & $4-30$ & $216.83 \pm 90.05$ & $4-20$ & & \\
\hline Siganus luridus & & & $0.17 \pm 0.12$ & $6-8$ & & \\
\hline \multicolumn{7}{|l|}{ Sparidae } \\
\hline Acanthopagrus bifasciatus & $0.28 \pm 0.28$ & 7 & & & & \\
\hline Diplodus noct & $28.33 \pm 27.75$ & $8-12$ & $1.11 \pm 1.11$ & 9 & & \\
\hline Sparus aurata & $0.06 \pm 0.06$ & 16 & $0.06 \pm 0.06$ & 15 & & \\
\hline \multicolumn{7}{|l|}{ Sphyraenidae } \\
\hline Sphyraena flavicauda & $5.28 \pm 3.62$ & $15-17$ & & & & \\
\hline \multicolumn{7}{|l|}{ Tetraodontiformes } \\
\hline \multicolumn{7}{|l|}{ Balistidae } \\
\hline Rhinecanthus assasi & & & $0.06 \pm 0.06$ & 6 & & \\
\hline \multicolumn{7}{|l|}{$\begin{array}{l}\text { Rninecanthus assast } \\
\text { Monacanthidae }\end{array}$} \\
\hline Aluterus scriptus & $0.11 \pm 0.11$ & 20 & & & & \\
\hline \multicolumn{7}{|l|}{ Ostraciidae } \\
\hline Ostracion cubicus & $0.39 \pm 0.18$ & $28-32$ & $0.67 \pm 0.21$ & $30-40$ & & \\
\hline \multicolumn{7}{|l|}{ Tetraodontidae } \\
\hline Arothron hispidus & $0.94 \pm 0.24$ & $15-40$ & $1.17 \pm 0.34$ & $30-40$ & & \\
\hline Arothron stellatus & $0.11 \pm 0.11$ & 30 & & & & \\
\hline \multicolumn{7}{|l|}{ Scorpaeniformes } \\
\hline \multicolumn{7}{|l|}{ Scorpaenidae } \\
\hline Pterois miles & & & & & $0.17 \pm 0.17$ & 15 \\
\hline \multicolumn{7}{|l|}{ Syngnathiformes } \\
\hline \multicolumn{7}{|l|}{ Fistulariidae } \\
\hline Fistularia commersonii & $0.06 \pm 0.06$ & 55 & & & $0.06 \pm 0.06$ & 40 \\
\hline
\end{tabular}

cies which were reported at the Eilat coral reef reserves (Table 3 ).

Overall, the abundances and biomass of wild fish were greater at the sea cages than at the open-water reference sites at both farms. Among these, the Carangidae (5 species), Sparidae (3 species), and Pomacentridae (3 species) were the 3 groups that contributed most to species richness. The diversity of wild fishes at both farms was greater than the diversity at the respective reference sites (Fig. 3, Table 4).

\section{Univariate analyses}

Abundances, biomasses, and number of species of wild fish were all significantly greater near fish cages than at reference sites, at both farms ( $p<0.05)$.

There were differences in abundance, biomass, and number of species between the fish farms and the reference sites (Table 5). Post hoc StudentNewman-Keuls (SNK) tests indicated that abundances were greater at Dagsuf (mean $\pm \mathrm{SE}=$ 
Table 3. List of fish species observed (listed in the same order as Table 2). Trophic tendency (TR; $\mathrm{C}=$ carnivore, $\mathrm{H}=\mathrm{herbivore}$, $\mathrm{O}=$ omnivore), trophic level $(\mathrm{TL}$, mean $\pm \mathrm{SD})$, environment $(\mathrm{P}=$ pelagic, $\mathrm{RA}=$ reef-associated), phylogenetic diversity index $\left(\mathrm{PD}_{50}\right)$, and feeding mode $(\mathrm{V}=$ variable, $\mathrm{SPF}=$ selective plankton feeding, $\mathrm{BS}=$ browsing on substrate, $\mathrm{GAP}=$ grazing on aquatic plants), were taken from Froese \& Pauly (2012). Note: all fish species are of commercial value, except Apogon fleurieu, Neopomacentrus miryae, and Arothron stellatus

\begin{tabular}{|c|c|c|c|c|c|c|}
\hline Fish species & Common name & TR & $\mathrm{TL}$ & Environment & $\mathrm{PD}_{50}$ & Feeding mode \\
\hline Atherinomorus lacunosus & Hardyhead silverside & $\mathrm{C}$ & $3.28 \pm 0.47$ & RA & 0.5005 & SPF \\
\hline Tylosurus choram & Red Sea houndfish & $\mathrm{C}$ & $4.39 \pm 0.79$ & $\mathrm{P}$ & 0.5156 & $\mathrm{P}$ \\
\hline Hyporhamphus gamberur & Red Sea halfbeak & $\mathrm{C}$ & $3.02 \pm 0.30$ & RA & 0.5000 & $P$ \\
\hline Ctenochaetus striatus & Striated surgeonfish & $\mathrm{H}$ & $2.00 \pm 0.00$ & RA & 0.5020 & BS \\
\hline Apogon fleurieu & Bullseye cardinalfish & $\mathrm{C}$ & $3.63 \pm 0.49$ & RA & 0.5000 & $\mathrm{P}$ \\
\hline Petroscirtes mitratus & Floral blenny & $\mathrm{H}$ & $2.00 \pm 0.00$ & RA & 0.5005 & GAP \\
\hline Caesio suevica & Suez fusilier & $\mathrm{C}$ & $3.40 \pm 0.45$ & RA & 0.5039 & $\mathrm{SPF}$ \\
\hline Atule mate & Yellowtail scad & $\mathrm{C}$ & $4.45 \pm 0.79$ & RA & 1.0000 & $\mathrm{P}$ \\
\hline Carangoides ferdau & Blue trevally & $\mathrm{C}$ & $4.50 \pm 0.80$ & RA & 0.5000 & $\mathrm{P}$ \\
\hline Carangoides fulvoguttatus & Yellowspotted trevally & $\mathrm{C}$ & $4.39 \pm 0.78$ & RA & 0.5000 & $\mathrm{P}$ \\
\hline Carangoides plagiotaenia & Barcheek trevally & $\mathrm{C}$ & $4.03 \pm 0.67$ & RA & 0.5000 & $\mathrm{P}$ \\
\hline Decapterus macarellus & Mackerel scad & $\mathrm{C}$ & $3.40 \pm 0.45$ & $\mathrm{P}$ & 0.5002 & $\mathrm{P}$ \\
\hline Chaetodon fasciatus & Diagonal butterflyfish & $\mathrm{O}$ & $3.34 \pm 0.61$ & RA & 0.5000 & $\mathrm{~V}$ \\
\hline Heniochus diphreutes & False moorish idol & $\mathrm{C}$ & $3.40 \pm 0.45$ & RA & 0.5039 & $\mathrm{SPF}$ \\
\hline Cirrhitichthys oxycephalus & Coral hawkfish & $\mathrm{C}$ & $4.01 \pm 0.66$ & RA & 0.5039 & $\mathrm{P}$ \\
\hline Thalassoma lunare & Moon wrasse & $\mathrm{C}$ & $3.48 \pm 0.56$ & RA & 0.5000 & $\mathrm{~V}$ \\
\hline Thalassoma rueppellii & Klunzinger's wrasse & $\mathrm{C}$ & $3.50 \pm 0.37$ & RA & 0.5000 & $\mathrm{P}$ \\
\hline Mulloides flavolineatus & Yellowstripe goatfish & $\mathrm{C}$ & $3.27 \pm 0.39$ & RA & 0.5156 & $\mathrm{P}$ \\
\hline Scolopsis ghanam & Arabian monocle bream & $\mathrm{C}$ & $3.62 \pm 0.51$ & RA & 0.5000 & $\mathrm{P}$ \\
\hline Abudefduf vaigiensis & Indo-Pacific sergeant & $\mathrm{O}$ & $2.59 \pm 0.35$ & RA & 0.5000 & GAP \\
\hline Neopomacentrus miryae & Miry's demoiselle & $\mathrm{C}$ & $3.40 \pm 0.45$ & RA & 0.5000 & SPF \\
\hline Pomacentrus trichourus & Paletail damsel & $\mathrm{C}$ & $2.68 \pm 0.30$ & RA & 0.5000 & SPF \\
\hline Auxis rochei rochei & Bullet tuna & $\mathrm{C}$ & $4.13 \pm 0.61$ & $\mathrm{P}$ & 0.7500 & $\mathrm{P}$ \\
\hline Scomber japonicus & Chub mackerel & $\mathrm{C}$ & $3.09 \pm 0.43$ & $\mathrm{P}$ & 0.5625 & $\mathrm{P}$ \\
\hline Anthias taeniatus & Red Sea anthaes & $\mathrm{C}$ & $3.40 \pm 0.45$ & RA & 0.5000 & $\mathrm{P}$ \\
\hline Pseudanthias squamipinnis & Sea goldie & $\mathrm{C}$ & $3.40 \pm 0.45$ & RA & 0.5000 & SPF \\
\hline Siganus rivulatus & Marbled spinefoot & $\mathrm{H}$ & $2.00 \pm 0.00$ & RA & 0.5000 & BS \\
\hline Siganus luridus & Dusky spinefoot & $\mathrm{H}$ & $2.00 \pm 0.02$ & RA & 0.5000 & BS \\
\hline Acanthopagrus bifasciatus & Twobar seabream & $\mathrm{C}$ & $3.39 \pm 0.43$ & RA & 0.5005 & $\mathrm{P}$ \\
\hline Diplodus noct & Red Sea seabream & $\mathrm{O}$ & $2.74 \pm 0.25$ & RA & 0.5001 & $\mathrm{~V}$ \\
\hline Sparus aurata & Gilthead seabream & $\mathrm{C}$ & $3.26 \pm 0.48$ & RA & 1.0000 & $\mathrm{P}$ \\
\hline Sphyraena flavicauda & Yellowtail barracuda & $\mathrm{C}$ & $3.76 \pm 0.60$ & RA & 0.5000 & $\mathrm{P}$ \\
\hline Rhinecanthus assasi & Picasso triggerfish & $\mathrm{C}$ & $3.50 \pm 0.37$ & RA & 0.5078 & $\mathrm{P}$ \\
\hline Aluterus scriptus & Scrawled filefish & $\mathrm{O}$ & $2.76 \pm 0.45$ & RA & 0.5312 & $\mathrm{~V}$ \\
\hline Ostracion cubicus & Yellow boxfish & $\mathrm{O}$ & $3.37 \pm 0.48$ & RA & 0.5020 & $\mathrm{~V}$ \\
\hline Arothron hispidus & White-spotted puffer & $\mathrm{O}$ & $3.11 \pm 0.27$ & RA & 0.5000 & $\mathrm{~V}$ \\
\hline Arothron stellatus & Starry toadfish & $\mathrm{O}$ & $3.34 \pm 0.46$ & RA & 0.5000 & $\mathrm{P}$ \\
\hline Pterois miles & Devil firefish & $\mathrm{C}$ & $3.73 \pm 0.63$ & RA & 0.5020 & $\mathrm{P}$ \\
\hline Fistularia commersonii & Bluespotted cornetfish & $\mathrm{C}$ & $4.26 \pm 0.74$ & RA & 0.6250 & $\mathrm{P}$ \\
\hline
\end{tabular}

$2702.4 \pm 295.8)$ than at Ardag and reference sites. Calculated biomass was also significantly higher at Dagsuf $(82143.9 \pm 16088.3 \mathrm{~kg})$, but the number of wild fish species was almost identical at the 2 farm sites, with $7.6 \pm 0.6$ and $7.1 \pm 0.7$ at Dagsuf and Ardag, respectively.

\section{Multivariate community analyses}

A dendrogram compiled from a Bray-Curtis similarity analysis of fish abundances shows that one distinct group, fish farm (similarity 64.66\%), is significantly different from the abundances at the reference sites (Fig. 4). Two-dimensional nMDS of fish abundances revealed a clear separation between the reference and Ardag sites (stress =0.01), but a less clear separation between Dagsuf and the reference site (stress $=0.01$ ). These differences were statistically demonstrated by the ANOSIM test. Pairwise ANOSIM comparisons between reference and sea-cage locations (Table 6) gave highly significant global $\mathrm{R}$ values for Ardag (comparison between reference and farm: $p=0.001$ ) and Dagsuf 

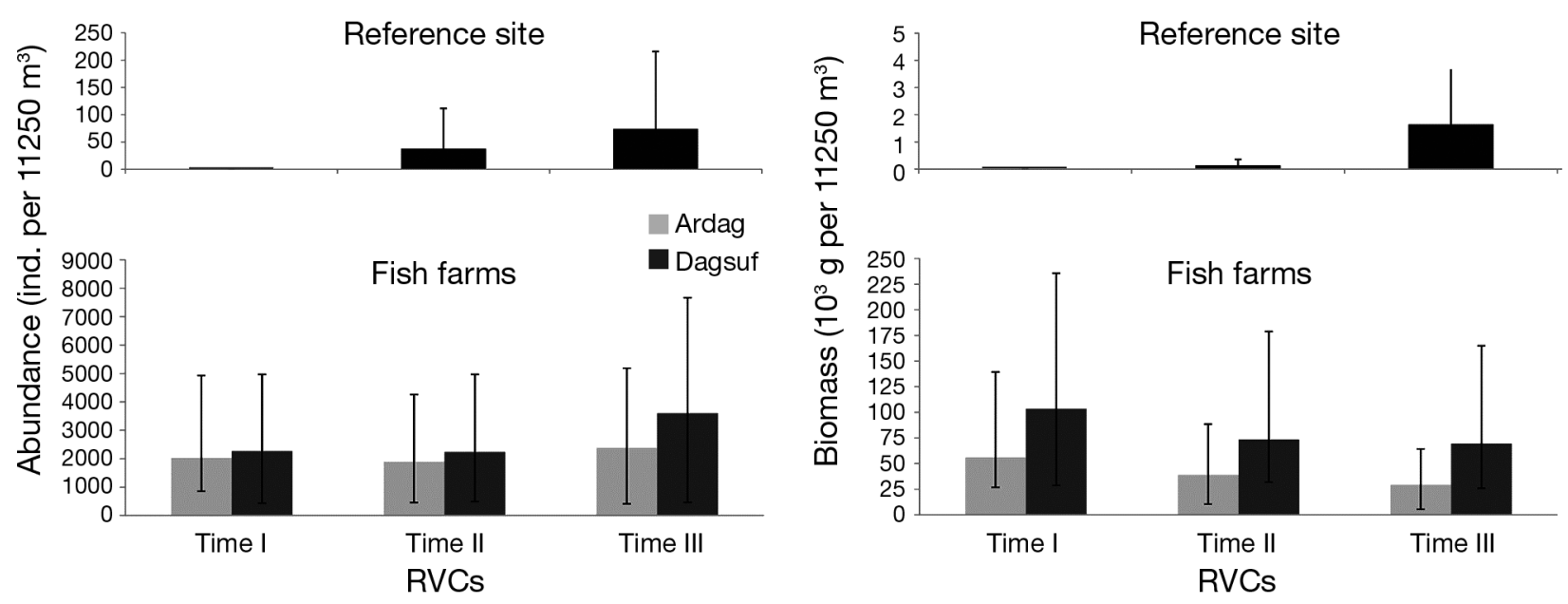

Fig. 3. Total abundance (mean \pm SE) (left panel) and biomass (mean \pm SE) (right panel) of coral reef-associated fish species at the reference site and fish farms on the 3 sampling dates ( $\mathrm{I}=$ August, $\mathrm{II}=$ September, $\mathrm{III}=$ October in 2007). RVC = rapid visual count

Table 4. ANOVA comparison among abundance, biomass, and number of species at the fish farms and reference sites on 3 dates nested within locations. Significant at ${ }^{* *} \mathrm{p} \leq 0.01 ;{ }^{* * *} \mathrm{p} \leq 0.001 ; \mathrm{ns}=$ no significant difference. NT $=$ no transformation performed

\begin{tabular}{|c|c|c|c|c|c|c|c|}
\hline & \multirow[t]{2}{*}{ df } & \multicolumn{2}{|c|}{ Abundance } & \multicolumn{2}{|c|}{ Biomass } & \multicolumn{2}{|c|}{ No. of species } \\
\hline & & MS & $F$ & MS & $F$ & MS & $F$ \\
\hline Location & 2 & 12100.84 & $205.62^{* * *}$ & 29927.51 & $48.67^{* * *}$ & 270.02 & $24.02^{* *}$ \\
\hline Time & 2 & 334.31 & $5.68^{\text {ns }}$ & 1950.40 & $3.17^{\mathrm{ns}}$ & 50.46 & $4.49^{\mathrm{ns}}$ \\
\hline Location $\times$ Time & 4 & 58.85 & $0.44^{\mathrm{ns}}$ & 614.90 & $0.27^{\mathrm{ns}}$ & 11.24 & $3.61^{* *}$ \\
\hline Residual & 45 & 134.32 & 2242.54 & 3.12 & & & \\
\hline Levene's test & & \multicolumn{2}{|c|}{0.06} & \multicolumn{2}{|c|}{0.00} & \multicolumn{2}{|c|}{0.016} \\
\hline Transformation & & \multicolumn{2}{|c|}{ Square root } & \multicolumn{2}{|c|}{ NT } & \multicolumn{2}{|c|}{ NT } \\
\hline
\end{tabular}

Table 5. Total abundance, biomass, Shannon-Weaver diversity, and number of species per $11250 \mathrm{~m}^{3}$ at fish farm and reference locations categorized by times of rapid visual counts (Times I to III, see Fig. 3). Values are mean \pm SE

\begin{tabular}{|llcccrc|}
\hline Site & & Count & Abundance & Biomass $(\mathrm{kg})$ & Diversity & No. of species \\
\hline Ardag & Farm & I & $2034.67 \pm 856.30$ & $56220.34 \pm 26898.66$ & $1.17 \pm 0.13$ & $6.00 \pm 0.63$ \\
& Reference & I & $0.00 \pm 0.00$ & $0.00 \pm 0.00$ & $0.00 \pm 0.00$ & $0.00 \pm 0.00$ \\
& Farm & II & $1899.00 \pm 460.62$ & $39089.91 \pm 10350.46$ & $1.29 \pm 0.21$ & $7.50 \pm 1.12$ \\
& Reference & II & $37.67 \pm 36.27$ & $136.75 \pm 80.77$ & $0.21 \pm 0.14$ & $0.83 \pm 0.40$ \\
& Farm & III & $2387.00 \pm 413.21$ & $29437.02 \pm 5379.82$ & $1.90 \pm 0.25$ & $9.17 \pm 0.98$ \\
& Reference & III & $74.00 \pm 67.72$ & $1643.37 \pm 1474.21$ & $0.19 \pm 0.15$ & $1.00 \pm 0.52$ \\
Dagsuf & Farm & & & & & \\
& Reference & I & $2267.00 \pm 434.53$ & $103477.11 \pm 28649.04$ & $0.82 \pm 0.22$ & $4.33 \pm 0.56$ \\
& Farm & I & $0.00 \pm 0.00$ & $0.00 \pm 0.00$ & $0.00 \pm 0.00$ & $0.00 \pm 0.00$ \\
& Reference & II & $2237.33 \pm 496.42$ & $73447.09 \pm 31823.59$ & $1.15 \pm 0.18$ & $6.33 \pm 0.61$ \\
& Farm & III & $3603.00 \pm 462.13$ & $69507.67 \pm 25855.43$ & $1.74 \pm 0.16$ & $10.50 \pm 0.67$ \\
& Reference & III & $74.00 \pm 67.72$ & $1643.37 \pm 1474.21$ & $0.19 \pm 0.15$ & $1.00 \pm 0.52$ \\
\hline
\end{tabular}

(comparison between reference and farm: $\mathrm{p}=$ 0.001), and also between Ardag and Dagsuf ( $\mathrm{p}=$ $0.001)$.

Further comparisons between sites performed by means of SIMPER analysis accentuated the relative contribution of fish species abundances to dissimilarities in the fish assemblages (Table 7). Noteworthy species that had a major impact on this were the highly abundant Atule mate and Neopomacentrus miryae. 


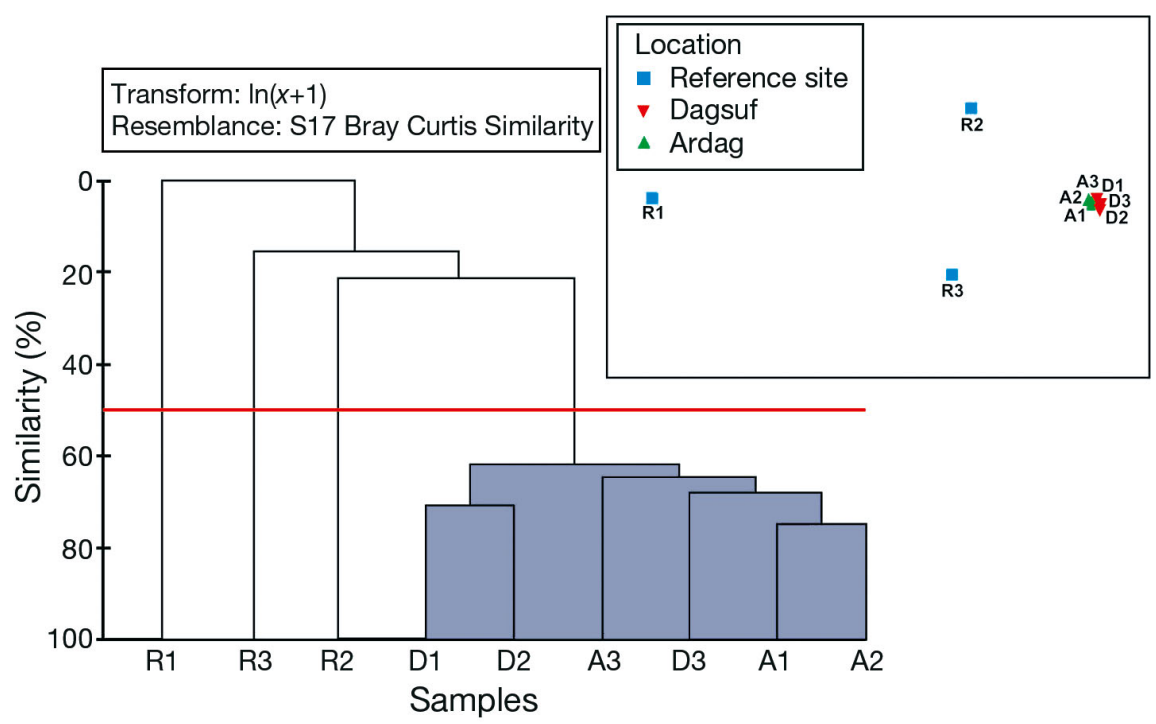

Fig. 4. Similarity dendrogram based on cluster analysis and non-metric multidimensional scaling (nMDS) plot ordination of fish species abundances associated with the fish farms ( $\mathrm{A}=$ Ardag; $\mathrm{D}=$ Dagsuf) and reference $(\mathrm{R})$ site on the 3 sampling dates in 2007 ( 1 = August, $2=$ September, $3=$ October, these numbers corresponding to Times I to III noted elsewhere)

Table 6. Pairwise analysis of similarity (ANOSIM) comparisons among reference, Ardag, and Dagsuf sites

\begin{tabular}{|lccccc|}
\hline Groups & R-value & $\begin{array}{c}\text { R significance } \\
\text { level (\%) }\end{array}$ & $\begin{array}{c}\text { Possible } \\
\text { permutations }\end{array}$ & $\begin{array}{c}\text { Actual } \\
\text { permutations }\end{array}$ & $\begin{array}{c}\text { No. } \\
\text { observed }\end{array}$ \\
\hline Ardag-Dagsuf & 0.296 & 20 & 10 & 10 & 2 \\
Ardag-Reference & 0.222 & 20 & 10 & 10 & 2 \\
Dagsuf-Reference & 0.222 & 10 & 10 & 10 & 1 \\
\hline
\end{tabular}

Table 7. Similarity percentage (SIMPER) procedure to test the degree of dissimilarity $(D)$ between farms and reference sites and between the 2 farm sites on the basis of the abundances of the dominant fish species

\begin{tabular}{|llc|}
\hline $\begin{array}{l}\text { Comparisons } \\
\text { between sites }\end{array}$ & Species & $\begin{array}{c}\text { Contribution } \\
\text { to dissimilarity (\%) }\end{array}$ \\
\hline Ardag-Reference & Atule mate & 13.66 \\
$(D=86.49)$ & Neopomacentrus miryae & 11.45 \\
& Siganus rivulatus & 11.10 \\
& Caesio suevica & 8.19 \\
Abudefduf vaigiensis & 8.02 \\
$(D=88.30)$ & Atule mate & 15.68 \\
& N. miryae & 10.79 \\
& C. suevica & 9.53 \\
Ardag-Dagsuf & Siganus rivulatus & 8.12 \\
$(D=36.84)$ & Scomber japonicus & 7.23 \\
& Scomber japonicus & 9.73 \\
& Tylosurus choram & 7.69 \\
& Hyporhamphus gamberur & 7.16 \\
& Sphyraena flavicauda & 6.64 \\
& Atherinomorus lacunosus & 6.13 \\
& Siganus rivulatus & 6.03 \\
\hline
\end{tabular}

\section{DISCUSSION}

Although aquaculture is not very new in the Red Sea, this is one of the first studies to describe the fishes that aggregate around net-cage fish farms in this region. The abundances, biomasses, and numbers of wild fish species were much greater adjacent to the 2 commercial fish farms studied than at nearby reference areas. Similar observations have been published in previous studies on wild fish around fish farms from different parts of the world, as reviewed above (see 'Introduction'). Moreover, a comparison of observed fish sizes to FishBase data (Froese \& Pauly 2012) suggests that more than half of the individuals recorded in the immediate vicinity of the fish cages were adults.

One of the surprising findings of the present study was the composition of the wild fish community. The most frequently observed species at the farms were Neopomacentrus miryae, Atule mate, Siganus rivulatus, Decapterus macarellus, Caesio suevica, Abudefduf vaigiensis, and Hyporhamphus gamberur - all usually coral-reef-associated species (Shpigel 1994). Although the net-cage fish farms were not situated near the coral reefs of Eilat or of Aqaba, 35 out of the 39 species observed at the farms were juveniles and adults of coral-reef fish species (Table 3 ). In a variety of studies on the movements of coral-reef fishes, including several studies conducted just south of the city of Eilat (Mazeroll \& Montgomery 1998, Jones et al. 2005, Claydon et al. 2012), most species did not exceed a range of $1 \mathrm{~km}$ from the reefs, whereas the fish farms in the present 
study were situated $>4 \mathrm{~km}$ from the nearest reef systems. It is also noteworthy that the composition of the fish community associated with the fish farms was not similar to the nearby sandy-shore community described by Golani et al. (2008).

Whereas larval and juvenile fishes often migrate great distances from the reefs they originated in (e.g. Palumbi 2003, McMahon et al. 2012), it is believed that the home range of the adults of these species is limited (Zeller et al. 2003). Although many reef species generally do not exceed a range of hundreds of meters from their home reefs, tagged individuals have been observed moving across much greater distances, occasionally in excess of several kilometers. It is assumed that such movements may be motivated by disturbance (e.g. habitat or predators), reproductive activities, and the pursuit of food, and limited by a variety of barriers (Appeldoorn et al. 2009). It is not clear what caused the reef fishes to migrate from their natural habitat to the Eilat fish farms, but the overwhelming numbers suggest that it was sufficient to overcome the barriers that normally prevent such movements.

In an earlier study, Angel et al. (2002) described a fish assemblage dominated by Neopomacentrus miryae and Pseudanthias squamipinnis associated with benthic artificial reefs that were deployed near the Ardag farm. Likewise, Sudirman et al. (2009) found several members from the nearby coral reefs when they examined the wild fish community associated with grouper cages in Indonesia. Although $>72 \%$ of the species we recorded were carnivores (Table 3 ), as defined by Bell \& Harmelin-Vivien (1983), several other trophic modes were observed in the unique fish-farm ecosystem. Atule mate, Caesio suevica, and Decapterus macarellus were all observed consuming other fishes around the fish cages, and many other carnivores, e.g. Fistularia commersonii, benefited from the presence of large schools of small fishes. Among the prey species, large schools of juvenile $N$. miryae and Siganus rivulatus were prevalent, and often found in close proximity to the net pens and components of the mooring systems, e.g. ropes, pipes, weights, etc. Abudefduf vaigiensis, Scomber japonicus, and Siganus rivulatus were all observed consuming uneaten feed pellets that fell through the cages, acting as omnivores. Dempster et al. (2002) and Sudirman et al. (2009) found similar behavior in their studies where different wild fish species fed on waste pellets and on fish feces.

Size class information indicated that $65 \%$ of the fish observed in the immediate vicinity of the fish farm swere adults. Of the 39 species, 35 were reef-associ- ated species, which were reported at the Eilat's coral reef reserves, and 34 were considered 'commercial' species. The majority of the observed species were trophically classified as carnivores (Table 3).

The persistent artificial food input and chemical attraction from farmed fishes probably influences which species of wild fishes associate with farms (Dempster et al. 2002). For example, carangids show particularly strong associations with FADs (Kingsford 1993) and with net-cage fish farms in the Mediterranean (Castro et al. 1999, Deudero et al. 1999). Two other groups that stand out in particular are the planktivorous pomacentrids and the siganids (Table 2). In addition to the aggregation of wild fish species, one of the ecological concerns regarding net-cage fish farms has to do with escapes. In their intensive surveys at numerous commercial fish farms, neither Boyra et al. (2004) nor Dempster et al. (2002) reported more than a few Sparus aurata and Dicentrarchus labrax escapees. Small escapes (10s to 100s of fish) are generally due to the loss of fishes during harvesting, whereas mass escapes $(10000 \mathrm{~s}$ to $100000 \mathrm{~s}$ of fish) are generally caused by storms, accidents, and poaching. In the present study, we observed only a few seabream/seabass escapees near the cage farms, and it is still unclear whether these introduced species have become established in the Red Sea.

Dempster et al. $(2004,2005)$ and Valle et al. (2007) emphasized that there are potentially negative ecological interactions between fish farms and wild fish populations, as a result of e.g. escapes and spreading of disease. Whereas these negative interactions may occur, there are some benefits that may be reaped as a result of wild fish aggregations. If the fish farms are turned into fish sanctuaries, with spatial protection for endangered wild fish stocks, due to the fact that they aggregate there, this may be regarded as an advantage. This idea may be extended to enable enhanced production of local fisheries through spillover of adults and increased spawning-stock biomass, which may consequently amplify larval recruitment, as described by Chiappone \& Sullivan (2000) and McClanahan \& Mangi (2000). A constant supply of uneaten fish feed to the wild fish population may enhance both growth and reproduction in this population while reducing the flux of organic matter to the sea floor and subsequent impacts on the benthos (Katz et al. 2002, Dempster et al. 2005, Bayle-Sempere et al. 2013). The diverse and colorful community of wild fishes and invertebrates present around fish farms, such as those observed in the Red Sea, may also provide an opportunity to develop highly attractive 
eco-tourism and agro-tourism, as described in Angel et al. (2002).

Due to the strong aggregative effect of fish farms, possible residence of fishes for periods of weeks to months, and the restrictions on fishing that apply within farm leasehold areas, we suggest that coastal net-cage fish farms may act as small marine protected areas. The reference sites used in the present study were situated only $500 \mathrm{~m}$ from each of the farms, yet they clearly showed that very few fish were present in waters adjacent to fish farms. This result is analogous to the association of reef fishes with artificial reefs, where a steep decline in abundance is typically observed at distances of just a few meters from the artificial structure (Bohnsack \& Sutherland 1985, Sanchez-Jerez \& Ramos-Espla 2000).

Acknowledgements. We are grateful to Steve Breitstein, Amir Yurman, Omer Polak, Nadav Shashar, Dan Tchernov, and other colleagues and students of the Inter University Institute (IUI) in Eilat for providing access to their laboratories. We also thank the staff of Ardag and Dagsuf farms for their assistance both underwater and above. This study was supported by the TUBITAK_BIDEB program.

\section{LITERATURE CITED}

Akyol O, Ertosluk O (2010) Fishing near sea-cage farms along the coast of the Turkish Aegean Sea. J Appl Ichthyol 26:11-15

Alston DE, Cabarcas-Nunez A, Helsley CE, Bridger C, Benetti D (2006) Standardized environmental monitoring of open ocean cage sites: basic considerations. World Aquacult 37:24-26

Angel DL, Spanier E (2002) An application of artificial reefs to reduce organic enrichment caused by net-cage fish farming: preliminary results. ICES J Mar Sci 59(Suppl): S324-S329

Angel DL, Eden N, Breitstein S, Yurman A, Katz T, Spanier E (2002) In situ biofiltration: a means to limit the dispersal of effluents from marine finfish cage aquaculture. Hydrobiologia 469:1-10

Appeldoorn RS, Aguilar-Perera A, Bouwmeester BLK, Dennis GD and others (2009) Movement of fishes (Grunts: Haemulidae) across the coral reef seascape: a review of scales, patterns and processes. Caribb J Sci 45:304-316

Atkinson MJ, Birk Y, Rosenthal H (2004) Evaluation of fish cages in the Gulf of Eilat. Report for the Ministries of Infrastructure, Environment and Agriculture, Israel

Bayle-Sempere JT, Arreguín-Sánchez F, Sanchez-Jerez P, Salcido-Guevara LA, Fernandez-Jover D, Zetina-Rejón MJ (2013) Trophic structure and energy fluxes around a Mediterranean fish farm. Ecol Modell 248:135-147

Bell JD, Harmelin-Vivien ML (1983) Fish fauna of French Mediterranean Posidonia oceanica meadows. 2. Feeding habits. Tethys 11:1-14

Benetti D, Brand L, Collins J, Orhun R and others (2006) Can offshore aquaculture of carnivorous fish be sustainable? World Aquacult 37:44-47

Beveridge M (2004) Cage aquaculture, 3rd edn. Blackwell Publishing, Oxford

Bjordal A, Skar AB (1992) Tagging of saithe (Pollachius virens L.) at a Norwegian fish farm: preliminary results on migration. ICES Counc Meet Pap 1992/G:35

Bohnsack JA, Sutherland DL (1985) Artificial reef research: a review with recommendations for future priorities. Bull Mar Sci 37:11-39

Bostock J, McAndrew B, Richards R, Jauncey K and others (2010) Aquaculture: global status and trends. Philos Trans R Soc Lond B 365:2897-2912

Boyra A, Sanchez-Jerez P, Tuya F, Espino F, Haroun R (2004) Attraction of wild coastal fishes to an Atlantic subtropical cage fish farm, Gran Canaria, Canary Islands. Environ Biol Fish 70:393-401

Bubic TS, Grubisic L, Ticina V, Katavic I (2011) Temporal and spatial variability of pelagic wild fish assemblages around Atlantic bluefin tuna Thunnus thynnus farms in the eastern Adriatic Sea. J Fish Biol 78:78-97

> Carss DN (1990) Concentration of wild and escaped fishes immediately adjacent to fish farm cages. Aquaculture 90: $29-40$

Castro J, Santiago J, Hernandez-Garcia V (1999) Fish associated with fish aggregation devices (FADs) off the Canary Islands (Central-East Atlantic). Sci Mar 63:191-198

Chiappone M, Sullivan S (2000) Marine reserve design criteria and measures of success: lessons learned from the Exuma Cays Land and Sea Park, Bahamas. Bull Mar Sci 66:691-705

> Clarke KR (1993) Non-parametric multivariate analyses of changes in community structure. Aust J Ecol 18:117-143

Clarke KR, Warwick RM (1994) Change in marine communities: an approach to statistical analysis and interpretation. Natural Environment Research Council, Plymouth

Claydon JAB, McCormick MI, Jones GP (2012) Patterns of migration between feeding and spawning sites in a coral reef surgeonfish. Coral Reefs 31:77-87

Costa-Pierce BA, Bridger CJ (2002) The role of aquaculture facilities as habitats and ecosystems. In: Stickney RR, McVey JP (eds) Responsible marine aquaculture. CAB International, Cambridge, p 105-144

Dempster T, Sanchez-Jerez P, Bayle-Sempere JT, GiménezCasalduero F, Valle C (2002) Attraction of wild fish to sea-cage fish farms in the south-western Mediterranean Sea: spatial and short-term temporal variability. Mar Ecol Prog Ser 242:237-252

Dempster T, Sanchez-Jerez P, Bayle-Sempere J, Kingsford M (2004) Extensive aggregation of wild fish at coastal sea-cage fish farms. Hydrobiologia 525:245-248

> Dempster T, Fernandez-Jover D, Sanchez-Jerez P, Tuya F, Bayle-Sempere J, Boyra A, Haroun RJ (2005) Vertical variability of wild fish assemblages around sea-cage fish farms: implications for management. Mar Ecol Prog Ser 304:15-29

> Deudero S, Merella P, Morales-Nin B, Massuti E, Alemany F (1999) Fish communities associated with FADs. J Fish Biol 63:199-207

FAO (2012) The state of world fisheries and aquaculture (SOFIA) 2012. FAO, Rome

Felsing M, Glenscross B, Telfer T (2005) Preliminary study on the effects of exclusion of wild fauna from aquaculture cages in a shallow marine environment. Aquaculture 243: $159-174$ 
Ferlin P, La Croix D (2000) Current state and future development of aquaculture in the Mediterranean region. World Aquacult 31:20-23

Freon P, Dagorn L (2000) Review of fish associative behaviour: toward a generalisation of the meeting point hypothesis. Rev Fish Biol Fish 10:183-207

Froese R, Pauly D (2012) FishBase. www.fishbase.org (Accessed 10 September 2012)

Golani D (2003) Fish assemblages associated with net pen mariculture and an adjacent rocky habitat in the Port of Ashdod, Israel (Eastern Mediterranean). Acta Adriat 44: 51-59

Golani D, Lerner A, Appelbaum-Golani B (2008) Fish biodiversity in the sandy shore of the Gulf of Eilat in close proximity to nearby fish farm activity. In: Por D (ed) AqabaEilat, the improbable gulf: environment, biodiversity and preservation. Magnes Press, Jerusalem, p 267-280

Halwart M, Soto D, Arthur JR (2007) Cage aquaculture: regional reviews and global overview. FAO Fish Tech Pap 498. FAO, Rome

Harmelin-Vivien ML, Harmelin JG, Chauvet C, Duval C and others (1985) Évaluation visuelle des peuplements et populations de poissons: méthodes et problèmes. Rev Ecol Terre Vie 40:467-539

Hunter JR, Mitchell CT (1967) Association of fishes with flotsam in the offshore waters of Central America. Fish Bull 66:13-29

Jones GP, Planes S, Thorrold SR (2005) Coral reef fish larvae settle close to home. Curr Biol 15:1314-1318

Katz T, Herut B, Genin A, Angel DL (2002) Gray mullets ameliorate organically enriched sediments below a fish farm in the oligotrophic Gulf of Aqaba (Red Sea). Mar Ecol Prog Ser 234:205-214

Kingsford M (1993) Biotic and abiotic structure in the pelagic environment: importance to small fish. Bull Mar Sci 53:393-415

Mazeroll AI, Montgomery WL (1998) Daily migrations of a coral reef fish in the Red Sea (Gulf of Aqaba, Israel): initiation and orientation. Copeia 1998:893-905

> McClanahan T, Mangi S (2000) Spillover of exploitable fishes from a marine park and its effect on the adjacent fishery. Ecol Appl 10:1792-1805

McMahon KW, Berumen ML, Thorrold SR (2012) Linking habitat mosaics and juvenile fish migration in a coral reef seascape. Proc Natl Acad Sci USA 109:15372-15376

Ogden JC, Quinn TP (1984). Migration in coral reef fishes: ecological significance. In: McCleave JD, Arnold GP, Dodson JJ, Neill WH (eds) Mechanisms of migration in fishes. Plenum Press, New York, NY, p 293-308

Palumbi SR (2003) Population genetics, demographic connectivity, and the design of marine reserves. Ecol Appl 13:146-158

Editorial responsibility: Catriona MacLeod,

Hobart, Tasmania, Australia
Phillips MJ, Beveridge MCM, Ross LG (1985) The environmental impact of salmonid cage culture on inland fisheries: present status and future trends. J Fish Biol 27(A): $123-127$

Reiss Z, Hottinger L (1984) The Gulf of Aqaba. Ecological micropaleontology. Ecol Stud 50. Springer-Verlag, Berlin

Sanchez-Jerez P, Ramos-Espla A (2000) Changes in fish assemblages associated with the deployment of an antitrawling reef in seagrass meadows. Trans Am Fish Soc 129:1150-1159

Sanchez-Mata A, Mora J (2000) A review of marine aquaculture in Spain: production, regulations and environmental monitoring. J Appl Ichthyol 16:209-213

Shpigel M (1994) Fish of the Red Sea. The Society for the Protection of Nature in Israel, Tel Aviv

Smith C, Machias A, Giannoulaki M, Somarakis S, Papadopoulou KN, Karakassis I (2003) Diversity study of wild fish fauna aggregating around fish farm cages by means of remotely operated vehicle (ROV). In: Abstr 7th Hel Symp Oceanogr Fish, Hersonissos, 6-9 May 2003, p 227

> Sudirman, Halide H, Jompa J, Zulfikar, Iswahyudin, McKinnon AD (2009) Wild fish associated with tropical sea cage aquaculture in South Sulawesi, Indonesia. Aquaculture 286:233-239

Theodorou J (1999) Greece focuses on marketing seabass and seabream. Seaf Int 14:35-36

Thetmeyer H, Pavlidis A, Cromey C (2003) Development of monitoring guidelines and modelling tools for environmental effects from Mediterranean aquaculture. Newsletter 3: Interactions between wild and farmed fish, p 7. Available at: www.meramed.com

> Tuya F, Boyra A, Sanchez-Jerez P, Haroun RJ (2005) Multivariate analysis of the bentho-demersal ichthyofauna along soft bottoms of the Eastern Atlantic: comparison between unvegetated substrates, seagrass meadows and sandy bottoms beneath sea-cage fish farms. Mar Biol 147: 1229-1237

Underwood AJ (1997) Experiments in ecology: their logical design and interpretation using analysis of variance. Cambridge University Press, Cambridge

> Valle C, Bayle-Sempere JT, Dempster T, Sanchez-Jerez P, Gimenez-Casalduero F (2007) Temporal variability of wild fish assemblages associated with a sea-cage farm in the south-western Mediterranean Sea. Estuar Coast Shelf Sci 72:299-307

Zakai D, Chadwick-Furman NE (2002) Impact of intensive recreational diving on reef corals at Eilat, Northern Red Sea. Biol Conserv 105:179-187

> Zeller D, Stoute SL, Russ GR (2003) Movements of reef fishes across marine reserve boundaries: effects of manipulating a density gradient. Mar Ecol Prog Ser 254:269-280

Submitted: March 6, 2013; Accepted: May 24, 2013

Proofs received from author(s): July 21, 2013 